\title{
Calculation of fermionic Green functions with Grassmann higher-order tensor renormalization group
}

\author{
Yusuke Yoshimura, ${ }^{1}$ Yoshinobu Kuramashi, ${ }^{1,2}$ Yoshifumi Nakamura, ${ }^{2,3}$ Shinji Takeda, ${ }^{4}$ and Ryo Sakai ${ }^{4}$ \\ ${ }^{1}$ Center for Computational Sciences, University of Tsukuba, Tsukuba, Ibaraki 305-8577, Japan \\ ${ }^{2}$ RIKEN Advanced Institute for Computational Science, Kobe, Hyogo 650-0047, Japan \\ ${ }^{3}$ Graduate School of System Informatics, Department of Computational Sciences, Kobe University, \\ Kobe, Hyogo 657-8501, Japan \\ ${ }^{4}$ Institute for Theorical Physics, Kanazawa University, Kanazawa 920-1192, Japan
}

(Received 24 November 2017; published 22 March 2018)

\begin{abstract}
We develop a calculational method for fermionic Green functions in the framework of Grassmann higher-order tensor renormalization group. The validity of the method is tested by applying it to the threedimensional free Wilson fermion system. We compare the numerical results for the chiral condensate and two-point correlation functions with the exact ones obtained by analytical methods.
\end{abstract}

DOI: 10.1103/PhysRevD.97.054511

\section{INTRODUCTION}

The tensor renormalization group (TRG) is a numerical renormalization group method in the tensor network scheme, which was originally introduced by Levin and Nave [1]. This method consists of two steps. We first make a tensor network representation for the target physical system. After that, we repeat the coarse-graining of the tensor network based on the singular value decomposition (SVD). The basic concept of this local transformation is similar to the block spin transformation [2]. An advantage of the TRG method is that it does not suffer from the sign problem inherent in the Monte Carlo methods. Subsequent to the appearance of TRG, it was extended for Grassmannvalued tensor networks, and the new method is called Grassmann TRG (GTRG) [3,4]. The TRG and GTRG methods have been applied to the two-dimensional relativistic quantum field theories in elementary particle physics: the lattice $\phi^{4}$ model [5], lattice Schwinger model $[4,6]$, and the finite-density lattice Thirring model [7]. Meanwhile, the TRG method itself has been improved by disentangling short-range correlations in tensor network renormalization (TNR) [8] and loop-TNR [9].

Although the original idea of TRG was restricted to twodimensional systems, Xie et al. invented a new method called higher-order TRG (HOTRG) with the use of higherorder SVD (HOSVD), which is formally applicable to any higher-dimensional system [10]. Its effectiveness is also

Published by the American Physical Society under the terms of the Creative Commons Attribution 4.0 International license. Further distribution of this work must maintain attribution to the author(s) and the published article's title, journal citation, and DOI. Funded by SCOAP ${ }^{3}$. demonstrated in Ref. [10] by applying it to two- and threedimensional Ising models. So far, the HOTRG method has been applied to several two- and three-dimensional systems: the three-dimensional Potts models [11], twodimensional XY model [12], two-dimensional O(3) model [13], and two-dimensional CP(1) model [14].

In order to use the tensor network scheme in lattice QCD, we need an algorithm which can be applied to fourdimensional relativistic fermion systems. The authors in Ref. [15] have formulated the GRASSMANN HOTRG (GHOTRG) based on the GTRG. It opened the way to higher-dimensional fermion systems but did not allow us to calculate physical quantities except the free energy and its differentiation. In this article, we develop a method for direct calculation of fermionic Green functions in the framework of GHOTRG and test the validity of the algorithm using the relativistic free Wilson fermion system, for which the exact values of Green functions are analytically calculable.

This paper is organized as follows. We make a brief review for the GHOTRG algorithm in Sec. II. In Sec. III, we explain the calculational method of fermionic Green functions with the GHOTRG method. Numerical results for Green functions in the relativistic free Wilson fermion system are presented in Sec. IV. Section V is devoted to the summary and outlook.

\section{GRASSMANN HIGHER-ORDER TENSOR RENORMALIZATION}

The detailed explanation for the GHOTRG algorithm is already given for the two-dimensional case in Ref. [15]. Although the extension to three-dimensional cases is rather straightforward, we give a brief review to fix the notations. We assume $a=1$ for the lattice units in the following. 


\section{A. Tensor network representation}

We first present a tensor network representation of the partition function for the three-dimensional free Wilson fermion with the Wilson parameter $r=1$ which is described in detail in Ref. [15]. The action with the fermion mass $m$ is given by

$$
\begin{gathered}
S=\sum_{n, n^{\prime}} \bar{\psi}_{n} D_{n, n^{\prime}} \psi_{n^{\prime}} \\
D_{n, n^{\prime}}=(m+3) \delta_{n, n^{\prime}}-\frac{1}{2} \sum_{\mu, \pm}\left(1 \pm \gamma_{\mu}\right) \delta_{n, n^{\prime} \pm \hat{\mu}}
\end{gathered}
$$

where $n=\left(n_{1}, n_{2}, n_{3}\right)$ is the lattice coordinate and the fermion fields $\psi_{n}, \bar{\psi}_{n}$ have two spinor components. A tensor network representation of the partition function is written by

$$
\begin{gathered}
Z=\int \mathcal{D} \psi \mathcal{D} \bar{\psi} e^{-S}=\sum_{\{x=0,1\}} \int \prod_{n} \mathcal{T}_{l_{n}}, \\
l_{n}=x_{1, n} \otimes x_{2, n} \otimes x_{3, n} \otimes x_{1, n-\hat{1}} \otimes x_{2, n-\hat{2}} \otimes x_{3, n-\hat{3}}
\end{gathered}
$$

where the indices are given by

$$
x_{i, n}=\left(x_{i, n, 1}, x_{i, n, 2}\right), \quad i=1,2,3,
$$

representing that $\psi$ has two spinor components. The tensor $\mathcal{T}$ is defined as

$$
\mathcal{T}_{l_{n}}=T_{l_{n}} G_{l_{n}},
$$

where the bosonic tensor $T$ have normal number elements whose explicit form is given in Ref. [15], and the Grassmann tensor $G$ is defined as

$$
\begin{array}{r}
G_{l_{n}}=\left(\prod_{i=1}^{3} d \bar{\eta}_{i, n, 2}^{x_{i, n, 2}} d \eta_{i, n, 1}^{x_{i, n, 1}}\right)\left(\prod_{i=1}^{3} d \eta_{i, n, 2}^{x_{i, n-\hat{i}, 2}} d \bar{\eta}_{i, n, 1}^{x_{i, n} \hat{i}, 1}\right) \\
\times \prod_{i=1}^{3}\left(\bar{\eta}_{i, n+\hat{i}, 1} \eta_{i, n, 1}\right)^{x_{i, n, 1}\left(\bar{\eta}_{i, n, 2} \eta_{i, n+\hat{i}, 2}\right)^{x_{i, n, 2}}} \\
\quad \int d \eta_{i, n, s} \eta_{i, n, s}=\int d \bar{\eta}_{i, n, s} \bar{\eta}_{i, n, s}=1 .
\end{array}
$$

The original fermion fields $\psi, \bar{\psi}$ have already been integrated out, while another set of independent Grassmann variables $\eta, \bar{\eta}$ have been introduced. It should be noted that the bosonic and the Grassmann tensors are not independent but they share a part of the indices of $l_{n}$. So they cannot be renormalized independently. Because of the Grassmann nature, elements of the bosonic tensor take nontrivial values only when the indices satisfy the following condition:

$$
\left[\sum_{i=1}^{3} \sum_{s=1}^{2}\left(x_{i, n, s}+x_{i, n-\hat{i}, s}\right)\right] \bmod 2=0 .
$$

Otherwise the element is zero. This allows us the block diagonalization of the bosonic tensors explained in the Appendix, which contributes to save the computational and memory costs.

\section{B. Renormalization of bosonic tensor}

We explain the renormalization procedure of bosonic tensors, which is mostly based on the original HOTRG [10]. Here we focus on the coarse-graining along the $\hat{1}$ direction depicted in Fig. 1. The coarse-graining for the other directions can be done in a similar way.

We first make up a contracted tensor $M$ by

$$
\begin{gathered}
M_{m_{n}}=\sum_{x_{1, n}} T_{l_{n+1}} T_{l_{n}} \sigma_{l_{n+\hat{1}} l_{n}}, \\
m_{n}=x_{1, n+\hat{1}} \otimes x_{2, n}^{+} \otimes x_{3, n}^{+} \otimes x_{1, n-\hat{1}} \otimes x_{2, n}^{-} \otimes x_{3, n}^{-}
\end{gathered}
$$

where the integrated indices are defined as

$$
\begin{gathered}
x_{i, n}^{+}=x_{i, n} \otimes x_{i, n+\hat{1}}, \\
x_{i, n}^{-}=x_{i, n-\hat{i}} \otimes x_{i, n+\hat{1}-\hat{i}}
\end{gathered}
$$

for $i=2,3 . \sigma$ is a sign factor originating from anticommutation properties in the renormalization of Grassmann part, which is explained in the next subsection.

Next we apply the eigenvalue decomposition to $\left(M M^{\dagger}\right)^{+}$ and $\left(M M^{\dagger}\right)^{-}$defined as

$$
\begin{aligned}
& \left(M M^{\dagger}\right)_{x_{2, n}^{+}, x_{2, n}^{+\prime}}^{+}=\sum_{a, c, d, e, f} M_{a x_{2, n}^{+} c d e f} M_{a x_{2, n}^{+\prime} c d e f}^{*}, \\
& \left(M M^{\dagger}\right)_{x_{2, n}^{-}, x_{2, n}^{-\prime}}^{-}=\sum_{a, b, c, d, f} M_{a b c d x_{2, n}^{-} f} M_{a b c d x_{2, n}^{-\prime} f}^{*}
\end{aligned}
$$

and obtain a unitary matrix $U^{ \pm}$and the corresponding eigenvalues $\lambda^{ \pm}$:

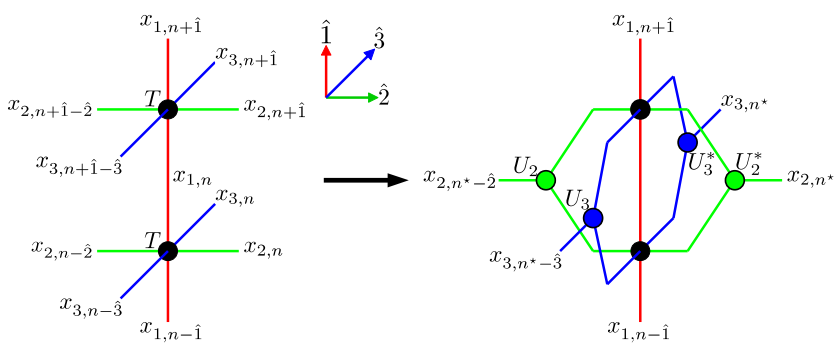

FIG. 1. Coarse-graining along the $x_{1}$ direction. 


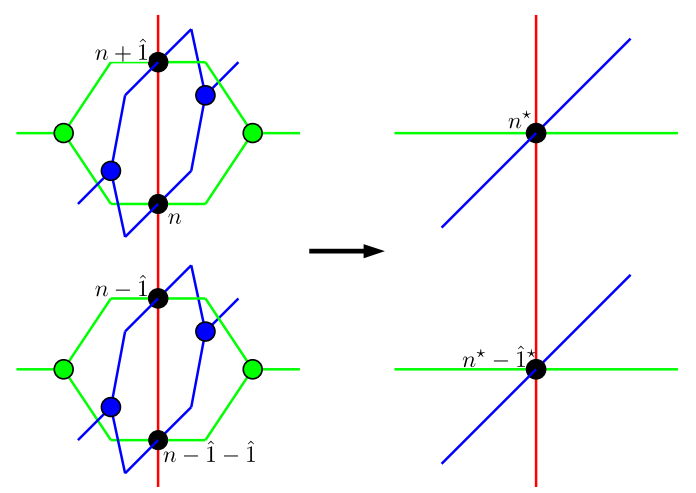

FIG. 2. Update of indices from $n$ to $n^{\star}$.

$$
\left(M M^{\dagger}\right)_{x_{2, n}^{ \pm}, x_{2, n}^{ \pm \prime}}^{ \pm}=\sum_{k}\left(U^{ \pm}\right)_{x_{2, n}^{ \pm} k} \lambda_{k}^{ \pm}\left(U^{ \pm}\right)_{x_{2, n}^{ \pm \prime}}^{*}
$$

Then, while assuming that $\lambda_{i}^{ \pm}$are obtained in descending order, we define $\epsilon^{ \pm}$as

$$
\epsilon^{ \pm}=\frac{\sum_{i>D_{\mathrm{cut}}} \lambda_{i}^{ \pm}}{\sum_{i} \lambda_{i}^{ \pm}}
$$

with a given $D_{\text {cut }}$ that one can choose. If $\epsilon^{+}<\epsilon^{-}$then $U_{2}=U^{+}$is adopted as an isometory to be used for the coarse-graining, and vice versa. In a similar manner, an isometory $U_{3}$ for the $\hat{3}$-direction is obtained. Restricting the new indices $1 \leq x_{2, n^{\star}}^{\mathrm{b}}, x_{3, n^{\star}}^{\mathrm{b}} \leq D_{\text {cut }}$, the coarse-grained bosonic tensor $T^{\star}$ is defined as a product of $U_{2}, U_{3}$, and $M$ :

$$
\begin{aligned}
T_{l_{n^{\star}}^{\star \mathrm{b}}}^{\star \mathrm{b}}= & \sum_{x_{2, n}^{ \pm}, x_{3, n}^{ \pm}} M_{m_{n}} \prod_{i=2}^{3}\left(U_{i}\right)_{i_{i, n}^{+} x_{i, n^{\star}}^{\mathrm{b}}}^{*}\left(U_{i}\right)_{x_{i, n^{-}} x_{i, n^{\star}-\hat{i}}^{\mathrm{b}},}, \\
l_{n^{\star}}^{\mathrm{b}}= & x_{1, n^{\star}} \otimes x_{2, n^{\star}}^{\mathrm{b}} \otimes x_{3, n^{\star}}^{\mathrm{b}} \\
& \otimes x_{1, n^{\star}-\hat{1}^{\star}} \otimes x_{2, n^{\star}-\hat{2}}^{\mathrm{b}} \otimes x_{3, n^{\star}-\hat{3}}^{\mathrm{b}} .
\end{aligned}
$$

For the lattice coordinate of the index $l$ for the tensor, here we unify $n, n+\hat{1}$ to $n^{\star}$ and define the new unit vector as $\hat{1}^{\star}=\hat{1}+\hat{1}$ as shown in Fig. 2 .

\section{Renormalization of Grassmann part}

According to the redefinition of the indices $x_{2, n}, x_{3, n}$ in the previous subsection, the Grassmann variables associated with them should be integrated out and replaced by new ones. We now explain the renormalization procedure of the Grassmann part focusing on the coarse-graining along the $\hat{1}$ direction. Note that the local sign factor may arise in dealing with the Grassmann variables because of the Grassmann nature, though the partition function itself is not changed.

We first extract the Grassmann parts:

$$
\begin{aligned}
& G_{l_{n+1}} G_{l_{n}}=\left(\prod_{i=1}^{3} d \bar{\eta}_{i, n+\hat{1}, 2}^{x_{i, n+\hat{1}, 2}} d \eta_{i, n+\hat{1}, 1}^{x_{i, n+1,1}}\right)\left(\prod_{i=1}^{3} d \eta_{i, n+\hat{1}, 2}^{x_{i, n+\hat{i}, 2}} d \bar{\eta}_{i, n+\hat{1}, 1}^{x_{i, n+\hat{i}, 1}}\right)\left(\prod_{i=1}^{3} d \bar{\eta}_{i, n, 2}^{x_{i, n, 2}} d \eta_{i, n, 1}^{x_{i, n}}\right)\left(\prod_{i=1}^{3} d \eta_{i, n, 2}^{x_{i, n}} d \bar{\eta}_{i, n, 1}^{x_{i, n-\hat{i}, 1}}\right) \\
& \times \prod_{i=1}^{3}\left(\bar{\eta}_{i, n+\hat{1}+\hat{i}, 1} \eta_{i, n+\hat{1}, 1}\right)^{x_{i, n+\hat{1}, 1}}\left(\bar{\eta}_{i, n+\hat{1}, 2} \eta_{i, n+\hat{1}+\hat{i}, 2}\right)^{x_{i, n+\hat{1}, 2}}\left(\bar{\eta}_{i, n+\hat{i}, 1} \eta_{i, n, 1}\right)^{x_{i, n, 1}}\left(\bar{\eta}_{i, n, 2} \eta_{i, n+\hat{i}, 2}\right)^{x_{i, n, 2}} .
\end{aligned}
$$

Next, in order to control sign factors from the anticommutation of Grassmann numbers on other sites, we introduce a new index and a new set of Grassmann variables satisfying

$$
\left(d \eta_{i, n^{\star}-\hat{i}} d \bar{\eta}_{i, n^{\star}} \bar{\eta}_{i, n^{\star}} \eta_{i, n^{\star}-\hat{i}}\right)^{x_{i, n^{\star}-\hat{i}}^{\mathrm{f}}}=1, \quad i=2,3,
$$

where new indices are defined as

$$
x_{i, n^{\star}-\hat{i}}^{\mathrm{f}}=\left[\sum_{s=1}^{2}\left(x_{i, n-\hat{i}, s}+x_{i, n+\hat{1}-\hat{i}, s}\right)\right] \bmod 2 .
$$

By inserting Eq. (21) into Eq. (20) and integrating $\bar{\eta}_{1, n+\hat{1}, 1}, \eta_{1, n, 1}, \bar{\eta}_{1, n, 2}$ and $\eta_{1, n+\hat{1}, 2}$, we obtain

$$
\begin{aligned}
& (-1)^{x_{1, n, 1}\left(1+x_{i, n, 2}\right)+\left(x_{2, n+\hat{1}-\hat{2}, 1}+x_{2, n+\hat{1}-\hat{2}, 2}\right)\left(x_{3, n-\hat{3}, 1}+x_{3, n-\hat{3}, 2}\right)}\left(\prod_{i=1}^{3} d \bar{\eta}_{i, n+\hat{1}, 2}^{x_{i, n+\hat{n}, 2}} d \eta_{i, n+\hat{1}, 1}^{x_{i, n+1}}\right)\left(\prod_{i=2}^{3} d \bar{\eta}_{i, n, 2}^{x_{i, n}} d \eta_{i, n, 1}^{x_{i, n, 1}}\right) \\
& \times d \eta_{1, n, 2}^{x_{1, n-\hat{1}, 2}} d \bar{\eta}_{1, n, 1}^{x_{1, n}-\hat{1}, 1}\left[\prod_{i=2}^{3} d \eta_{i, n, 2}^{x_{i, n-\hat{i}, 2}} d \bar{\eta}_{i, n, 1}^{x_{i, n-\hat{i}, 1}} d \eta_{i, n+\hat{1}, 2}^{x_{i, n+\hat{1}}} d \bar{\eta}_{i, n+\hat{1}, 1}^{x_{i, n+\hat{1}-\hat{i}, 1}} d \eta_{i, n^{\star}-\hat{i}}^{x_{i, n^{\star}}^{\mathrm{f}} \hat{i}} d \bar{\eta}_{i, n^{\star}}^{x_{i, n^{\star}}^{\mathrm{f}}}\left(\bar{\eta}_{i, n^{\star}} \eta_{i, n^{\star}-\hat{i}}\right)^{x_{i, n^{\star}-\hat{i}}^{\mathrm{f}}}\right] \\
& \times\left[\prod_{i=1}^{3}\left(\bar{\eta}_{i, n+\hat{1}+\hat{i}, 1} \eta_{i, n+\hat{1}, 1}\right)^{x_{i, n+\hat{1}, 1}}\left(\bar{\eta}_{i, n+\hat{1}, 2} \eta_{i, n+\hat{1}+\hat{i}, 2}\right)^{x_{i, n+\hat{1}, 2}}\right]\left[\prod_{i=2}^{3}\left(\bar{\eta}_{i, n+\hat{i}, 1} \eta_{i, n, 1}\right)^{x_{i, n, 1}}\left(\bar{\eta}_{i, n, 2} \eta_{i, n+\hat{i}, 2}\right)^{x_{i, n, 2}}\right] .
\end{aligned}
$$


Thanks to Eq. (22), the combinations of Grassmann measures,

$$
d \eta_{i, n, 2}^{x_{i, n-\hat{i}, 2}} d \bar{\eta}_{i, n, 1}^{x_{i, n-\hat{i}, 1}} d \eta_{i, n+\hat{1}, 2}^{x_{i, n+\hat{i}-\hat{i}, 2}} d \bar{\eta}_{i, n+\hat{1}, 1}^{x_{i, n+\hat{i}-1,1}} d \eta_{i, n^{\star}-\hat{i}}^{x_{i, n^{\star}}^{\mathrm{f}}}
$$

for $i=2,3$ are Grassmann-even and thus we can now freely move it. By shifting the coordinate of the Grassmann measures and $\bar{\eta}_{2, n^{\star}} \eta_{2, n^{\star}-\hat{2}}, \bar{\eta}_{3, n^{\star}} \eta_{3, n^{\star}-\hat{3}}$, one finds that Eq. (23) turns out to be

$$
\begin{aligned}
& (-1)^{x_{1, n, 1}\left(1+x_{i, n, 2}\right)+\left(x_{2, n+\hat{1}-\hat{2}, 1}+x_{2, n+\hat{1}-\hat{2}, 2}\right)\left(x_{3, n-\hat{3}, 1}+x_{3, n-\hat{3}, 2}\right)+x_{2, n^{\star}}^{\mathrm{f}} x_{3, n}^{\mathrm{f}}} d \bar{\eta}_{1, n+\hat{1}, 2}^{x_{1, n+\hat{1}, 2}} d \eta_{1, n+\hat{1}, 1}^{x_{1, n+1,1}} \\
& \times\left\{\left(\prod_{i=2}^{3} d \bar{\eta}_{i, n+\hat{1}, 2}^{x_{i, n+1,2}} d \eta_{i, n+\hat{1}, 1}^{x_{i, n+\hat{1}, 1}}\right)\left(\prod_{i=2}^{3} d \bar{\eta}_{i, n, 2}^{x_{i, n, 2}} d \eta_{i, n, 1}^{x_{i, n, 1}}\right)\left(\prod_{i=2}^{3} d \eta_{i, n+\hat{i}, 2}^{x_{i, n}} d \bar{\eta}_{i, n+\hat{i}, 1}^{x_{i, n}} d \eta_{i, n+\hat{1}+\hat{i}, 2}^{x_{i, n+\hat{1}, 2}} d \bar{\eta}_{i, n+\hat{1}+\hat{i}, 1}^{x_{i, n+1,1}}\right)\right. \\
& \left.\times\left[\prod_{i=2}^{3}\left(\bar{\eta}_{i, n+\hat{1}+\hat{i}, 1} \eta_{i, n+\hat{1}, 1}\right)^{x_{i, n+\hat{1}, 1}}\left(\bar{\eta}_{i, n+\hat{1}, 2} \eta_{i, n+\hat{1}+\hat{i}, 2}\right)^{x_{i, n+\hat{1}, 2}}\left(\bar{\eta}_{i, n+\hat{i}, 1} \eta_{i, n, 1}\right)^{x_{i, n, 1}}\left(\bar{\eta}_{i, n, 2} \eta_{i, n+\hat{i}, 2}\right)^{x_{i, n, 2}}\right]\right\} \\
& \times\left(\prod_{i=2,3} d \eta_{i, n^{\star}}^{x_{i, n^{\star}}^{\mathrm{f}}}\right) d \eta_{1, n, 2}^{x_{1, n \hat{1}, 2}} d \bar{\eta}_{1, n, 1}^{x_{1, n-1,1}}\left(\prod_{i=2,3} d \bar{\eta}_{i, n^{\star}}^{x_{i, n^{\star}}^{\mathrm{f}}}\right) \\
& \times\left(\bar{\eta}_{1, n+\hat{1}+\hat{1}, 1} \eta_{1, n+\hat{1}, 1}\right)^{x_{1, n+\hat{1}, 1}}\left(\bar{\eta}_{1, n+\hat{1}, 2} \eta_{1, n+\hat{1}+\hat{1}, 2}\right)^{x_{1, n+\hat{1}, 2}} \prod_{i=2,3}\left(\bar{\eta}_{i, n^{\star}+\hat{i}} \eta_{i, n^{\star}}\right)^{x_{i, n^{\star}}^{\mathrm{f}}},
\end{aligned}
$$

where the Grassmann numbers in the braces can be integrated out. After the integration, the coarse-grained Grassmann part and the sign factor of Eq. (10) results in

$$
\begin{aligned}
& \sigma_{l_{n+1} l_{n}} G_{l_{n^{\star}}^{\mathrm{f}}}, \quad l_{n^{\star}}^{\mathrm{f}}=x_{1, n^{\star}} \otimes x_{2, n^{\star}}^{\mathrm{f}} \otimes x_{3, n^{\star}}^{\mathrm{f}} \otimes x_{1, n^{\star}-\hat{1}^{\star}} \otimes x_{2, n^{\star}-\hat{2}}^{\mathrm{f}} \otimes x_{3, n^{\star}-\hat{3}}^{\mathrm{f}},
\end{aligned}
$$

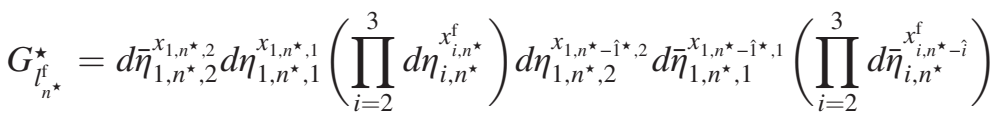

$$
\begin{aligned}
& \times\left(\bar{\eta}_{1, n^{\star}+\hat{1}^{\star}, 1} \eta_{1, n^{\star}, 1}\right)^{x_{1, n^{\star}, 1}}\left(\bar{\eta}_{1, n^{\star}, 2} \eta_{1, n^{\star}+\hat{1}^{\star}, 2}\right)^{x_{2, n^{\star}, 2}} \prod_{i=2}^{3}\left(\bar{\eta}_{i, n^{\star}+\hat{i}} \eta_{i, n^{\star}}\right)^{x_{i, n^{\star}}^{\mathrm{f}}},
\end{aligned}
$$

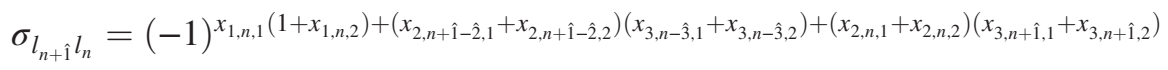

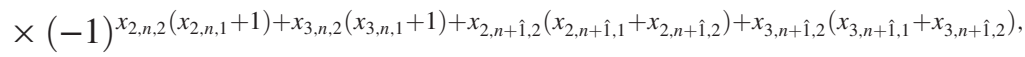

where the coordinates in $G$ are unified as in the previous subsection. Note that, from Eq. (9) and (22), a new constraint for the updated indices is given by

$$
p_{l_{n^{\star}}^{\mathrm{f}}} \equiv\left[\sum_{s=1}^{2}\left(x_{1, n^{\star}, s}+x_{1, n^{\star}-\hat{1}^{\star}, s}\right)+\sum_{i=2}^{3}\left(x_{i, n^{\star}}^{\mathrm{f}}+x_{i, n^{\star}-\hat{i}}^{\mathrm{f}}\right)\right] \bmod 2=0 .
$$

By combining the renormalization procedures for the bosonic and the Grassmann parts, the coarse-grained tensor is expressed as

$$
\mathcal{T}_{l_{n^{\star}}^{\star}}^{\star}=T_{l_{n^{\star}}^{\star}}^{\star} G_{l_{n^{\star}}^{\star}}^{\star}
$$

where the indices $l_{n^{\star}}$ are defined as

$$
l_{n^{\star}}=\left(l_{n^{\star}}^{\mathrm{b}}, l_{n^{\star}}^{\mathrm{f}}\right)
$$

and the coarse-grained bosonic tensor which has the new indices arise from the Grassmann part is given by

$$
T_{l_{n^{\star}}^{\star}}^{\star}=T_{l_{n^{\star}}^{\star}}^{\star, b} \delta_{p_{l^{\ddagger}}, 0}
$$

with the Kronecker delta to satisfy Eq. (28).

\section{CALCULATIONAL METHOD FOR FERMIONIC GREEN FUNCTIONS}

\section{A. Chiral condensate}

We first explain a method to calculate the chiral condensate defined as 


$$
\begin{gathered}
\langle\bar{\psi} \psi\rangle=\frac{Z_{I}}{Z} \\
Z_{I}=\frac{1}{V} \int \mathcal{D} \psi \mathcal{D} \bar{\psi} e^{-S}\left(\sum_{n, i} \bar{\psi}_{n, i} \psi_{n, i}\right)
\end{gathered}
$$

where $V$ is the system volume. Thanks to the translational invariance, Eq. (32) is rewritten as

$$
Z_{I}=\int \mathcal{D} \psi \mathcal{D} \bar{\psi} e^{-S}\left(\sum_{i} \bar{\psi}_{n_{0}, i} \psi_{n_{0}, i}\right) .
$$

Due to the presence of $\bar{\psi}_{n_{0}} \psi_{n_{0}}$, the bosonic tensor on the site $n_{0}$ is modified. Now we introduce the "impure tensor" and define the bosonic part in it as $I$. Then the impure tensor with the Grassmann part is defined as

$$
\mathcal{I}_{l_{n_{0}}}=I_{l_{n_{0}}} G_{l_{n_{0}}}
$$

Then Eq. (33) is expressed as

$$
Z_{I}=\sum_{\{x\}} \mathcal{I}_{l_{n_{0}}} \prod_{n \neq n_{0}} \mathcal{T}_{l_{n}}
$$

In the following, the renormalization algorithm is formulated based on this expression.

First of all, the renormalization procedure for the normal tensors is explained in Sec. II. On the other hand, in the case of the impure tensor, the contracted tensor of Eq. (10) is modified as

$$
M_{m_{n_{0}}}^{\text {impure }}=\sum_{x_{n_{0}}} T_{l_{n_{0}+1}} I_{l_{n_{0}}} \sigma_{l_{n_{0}+1} l_{n_{0}}}
$$

With the use of the isometries that are the same as in the normal tensors, we obtain the following new bosonic tensor:

$$
I_{\substack{l_{n_{0}^{\star}}^{\star} \\ \mathrm{b}}}^{\mathrm{b}}=\sum_{x_{2, n_{0}}^{ \pm}, x_{3, n_{0}}^{ \pm}} M_{m_{n_{0}}}^{\mathrm{impure}} \times \prod_{i=2}^{3}\left(U_{i}\right)_{x_{i, n_{0}}^{+} x_{i, n_{0}^{\star}}^{\mathrm{b}}}^{\mathrm{b}}\left(U_{i}\right)_{x_{i, n_{0}}^{-} x_{i, n_{0}^{\star}}^{\mathrm{b}}-\hat{i}^{*}}
$$

Figure 3 gives a schematic view of the coarse-graining procedure for the impure tensor. The renormalization procedure for the Grassmann part is similar to that for the normal tensors. Thus, the coarse-grained impure tensor is expressed as

$$
\mathcal{I}_{l_{n_{0}^{\star}}^{\star}}^{\star}=I_{l_{n_{0}^{\star}}^{\star}}^{\star} G_{l_{0}^{\star}}^{\star}
$$

\footnotetext{
${ }^{1}$ The coarse-graining for the impure tensor in the framework of TRG is discussed in Ref. [16,17].
}

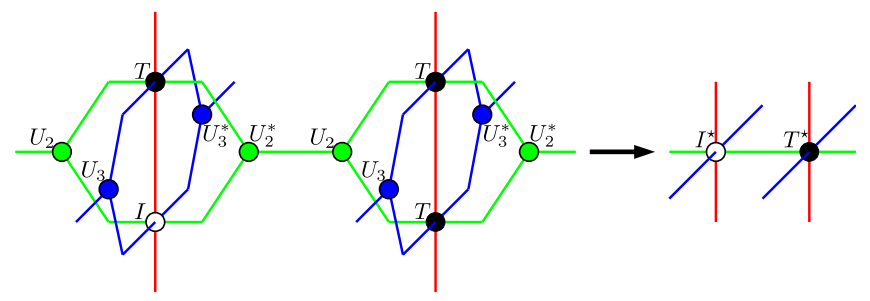

FIG. 3. Coarse-graining procedure for the impure tensor.

$$
I_{l_{n_{0}^{\star}}^{\star}}^{\star}=I_{l_{n_{0}^{\star}}^{\mathrm{b}}}^{\star \mathrm{b}} \delta_{p_{n_{0}^{\star}}^{\mathrm{f}}, 0} \cdot
$$

\section{B. Fermion two-point correlation functions}

We now explain a calculational method for the fermion two-point correlation function defined as

$$
C_{s_{1} s_{2}}\left(n_{1}, n_{2}\right)=\frac{Z_{s_{1} s_{2}}\left(n_{1}, n_{2}\right)}{Z}
$$

with

$$
Z_{s_{1} s_{2}}\left(n_{1}, n_{2}\right)=\int \mathcal{D} \psi \mathcal{D} \bar{\psi} e^{-S} \bar{\psi}_{n_{1}, s_{1}} \psi_{n_{2}, s_{2}}
$$

In this case, the tensors on the site $n_{1}$ and $n_{2}$ become the impure tensors, which are denoted by

$$
\begin{aligned}
& \overline{\mathcal{I}}_{l_{n_{1}}}=\bar{I}_{l_{n_{1}}} G_{l_{n_{1}}}, \\
& \mathcal{I}_{l_{n_{2}}}=I_{l_{n_{2}}} G_{l_{n_{2}}},
\end{aligned}
$$

respectively. Inclusion of odd numbers of fermions at the site $n_{1}$ and $n_{2}$ modifies the constraint described in Eq. (9) as

$$
\left[\sum_{i=1}^{3} \sum_{s=1}^{2}\left(x_{i, n, s}+x_{i, n-\hat{i}, s}\right)\right] \bmod 2=1 .
$$

Then Eq. (42) is expressed as

$$
Z_{s_{1} s_{2}}=\sum_{\{x\}} \overline{\mathcal{I}}_{l_{n_{1}}} \mathcal{I}_{l_{n_{2}}} \prod_{n \neq n_{1}, n_{2}} \mathcal{T}_{l_{n}}
$$

In the calculation of the fermion two-point correlation functions, the renormalization procedures are classified into three types according to the positions of the impure tensors. We explain them in order.

$$
\text { 1. } n_{1}+\hat{\mathbf{1}}=n_{2}
$$

In this case, two impure tensors are merged into one impure tensor as shown in Fig. 4. The contracted tensor is obtained by

$$
M_{m_{n_{1}}}^{\text {impure }}=\sum_{x_{1, n_{1}}} I_{l_{n_{2}}} \bar{I}_{l_{n_{1}}} \sigma_{l_{n_{2}} l_{n_{1}}}^{\prime}
$$




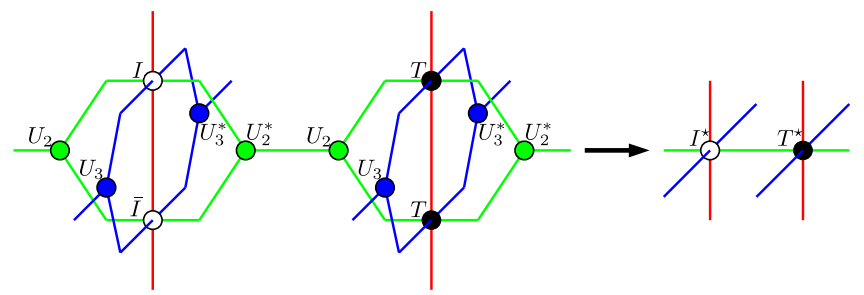

FIG. 4. Coarse-graining procedure for two impure tensors in the case of $n_{1}+\hat{1}=n_{2}$.

where $\sigma^{\prime}$ is the sign factor modified by Eq. (45) as follows:

$$
\sigma_{l_{n_{2}} l_{n_{1}}}^{\prime}=(-1)^{\sum_{i=2}^{3} \sum_{s=1}^{2} x_{i, n_{2}-\hat{i}, s}} \sigma_{l_{n_{2}} l_{n_{1}}} .
$$

We use the isometries which are the same as those for the normal tensors. After the two impure tensors are merged, we obtain a tensor network with a single impure tensor described in the previous subsection.

$$
\text { 2. } n_{1} \pm \hat{\mathbf{2}}=n_{2} \text { or } n_{1} \pm \hat{\mathbf{3}}=n_{2}
$$

We exemplify the case of $n_{1}+\hat{2}=n_{2}$ as depicted in Fig. 5. For each impure tensor, we construct the corresponding contracted tensor:

$$
\begin{aligned}
\bar{M}_{m_{n_{1}}}^{\text {impure }} & =\sum_{x_{1, n_{1}}} T_{l_{n_{1}+1}} \bar{I}_{l_{n_{1}}} \sigma_{l_{n_{1}+1} l_{n_{1}}}^{\prime}, \\
M_{m_{n_{2}}}^{\text {impure }} & =\sum_{x_{1, n_{2}}} T_{l_{n_{2}+1}} I_{l_{n_{2}}} \sigma_{l_{n_{2}+1} l_{n_{2}}}^{\prime} .
\end{aligned}
$$

The unitary matrices $U_{2}, U_{3}$ are obtained by the renormalization procedure for the normal tensors. In addition, we apply the eigenvalue decomposition to $\left(M M^{\dagger}\right)^{+}$and $\left(M M^{\dagger}\right)^{-}$defined by

$$
\begin{aligned}
& \left(M M^{\dagger}\right)_{x_{2, n_{1}}^{+}, x_{2, n_{1}}^{+}}^{\text {impure, }}=\sum_{a, c, d, e, f} \bar{M}_{a x_{2, n_{1}}^{+} c d e f}^{\text {impure }} \bar{M}_{a x_{2, n_{1}}^{+,} c d e f}^{\text {impure }}, \\
& \left(M M^{\dagger}\right)_{x_{2, n_{2}}^{-}, x_{2, n_{2}}^{-\prime}}^{\text {impure,- }}=\sum_{a, b, c, d, f} M_{a b c d x_{2, n_{2}} f}^{\text {impure }} M_{a b c d x_{2, n_{2}}^{-\prime} f}^{\text {impure }}
\end{aligned}
$$

and obtain a unitary matrix $U^{ \pm}$and the eigenvalues $\lambda^{ \pm}$and $\epsilon^{ \pm}$in the same manner as Eqs. (16) and (17). If $\epsilon^{+}<\epsilon^{-}$,

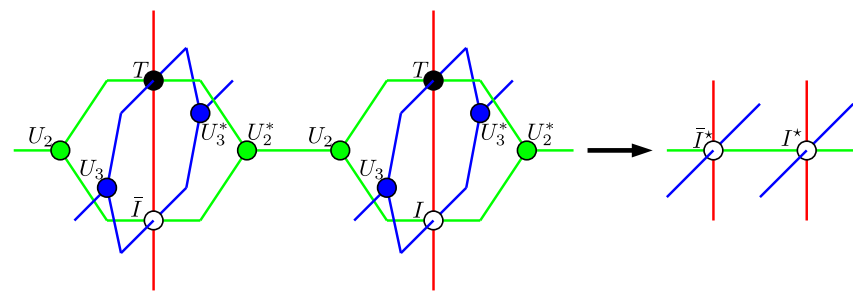

FIG. 5. Coarse-graining procedure for two impure tensors in the case of $n_{1} \pm \hat{2}=n_{2}$. then $W=U^{+}$is adopted, and vice versa. The new bosonic tensors $\bar{I}^{\star}, I^{\star}$ are obtained by using $U_{2}, U_{3}$ and $W$ :

$\bar{I}_{l_{n_{1}^{*}}^{\star}, \mathrm{b}}^{\star \mathrm{b}}=\sum_{x_{2, n_{1}}^{ \pm}, x_{3, n_{1}}^{ \pm}} \bar{M}_{m_{n_{1}}}^{\text {impure }} W_{x_{2, n_{1}}^{+} x_{2, n_{1}^{\star}}^{\mathrm{b}}}^{*}\left(U_{3}\right)_{x_{3, n_{1}}^{+} x_{3, n_{1}^{*}}^{\mathrm{b}}}^{*} \prod_{i=2}^{3}\left(U_{i}\right)_{x_{i, n_{1}}^{-} x_{i, n}^{\mathrm{b}} x_{1}^{\mathrm{b}}-\hat{i}}$,

$$
\begin{aligned}
I_{l_{n_{2}^{*}}^{\mathrm{b}}}^{\star \mathrm{b}}= & \sum_{x_{2, n_{1}}^{ \pm}, x_{3, n_{1}}^{ \pm}} M_{m_{n_{2}}}^{\text {impure }} \prod_{i=2}^{3}\left(U_{i}\right)_{x_{i, n_{1}}^{+} x_{i, n_{1}^{\star}}^{\mathrm{b}}} W_{x_{2, n_{2}}^{-} x_{2, n_{2}^{*}-\hat{2}}^{\mathrm{b}}} \\
& \times\left(U_{3}\right)_{x_{3, n_{2}}^{-} x_{3, n_{2}^{\star}-\hat{s}^{*}}^{\mathrm{b}}}
\end{aligned}
$$

The rest of the procedure is similar to that for the normal tensors.

\section{Other cases}

This is the case that two impure tensors are renormalized independently. The contracted tensors for impure tensors are given by

$$
\begin{aligned}
& \bar{M}_{m_{n_{1}}}^{\text {impure }}=\sum_{x_{1, n_{1}}} T_{l_{n_{1}+1}} \bar{I}_{l_{n_{1}}} \sigma_{l_{n_{1}+\hat{1}} l_{n_{1}}}^{\prime}, \\
& M_{m_{n_{2}}}^{\text {impure }}=\sum_{x_{1, n_{2}}} T_{l_{n_{2}+1}} I_{l_{n_{2}}} \sigma_{l_{n_{2}+\hat{1}} l_{n_{2}}}^{\prime} .
\end{aligned}
$$

The isometries are obtained by the renomalization procedure for the normal tensors. The rest of the procedure is similar to that for the normal tensors.

\section{NUMERICAL RESULTS}

In this section, we present the numerical results. The anti-periodic boundary condition is imposed for one of the directions. We employ it to test the calculational method of the fermionic Green functions on a $256^{3}$ lattice.

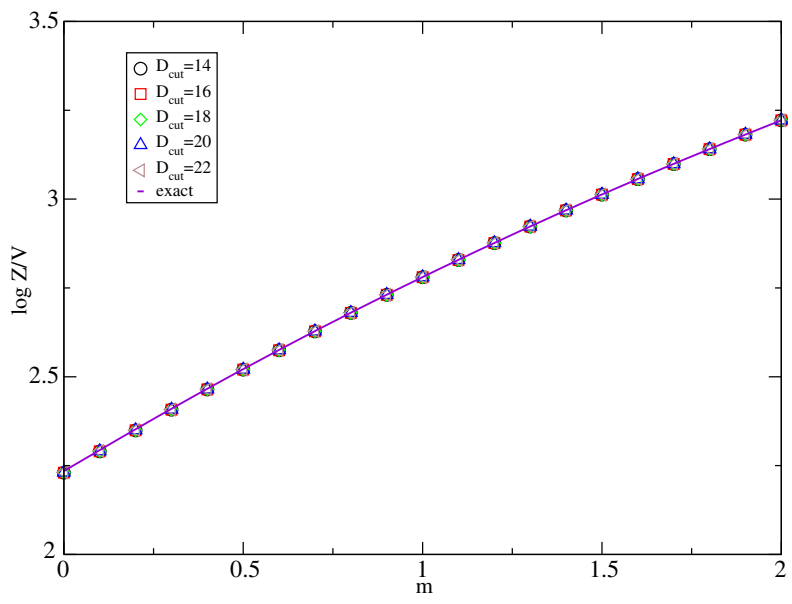

FIG. 6. Free energy as a function of $m$. 


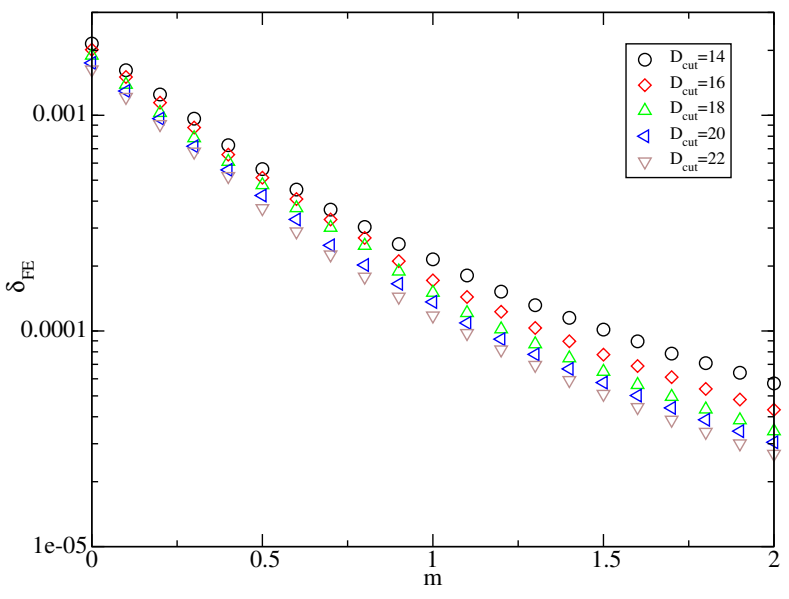

FIG. 7. Error of free energy defined by Eq. (57) as a function of $m$.

\section{A. Free energy}

We first present the results for the free energy of three-dimensional free Wilson fermion system. Figure 6 compares the numerical results with the exact values for the free energy as a function of the fermion mass $m$. The latter is obtained by an analytical calculation in the momentum spaces. It is hard to detect the deviation between the measured values with $D_{\text {cut }} \geq 14$ and the exact ones. In Fig. 7, we directly plot the magnitude of the error defined by

$$
\delta_{\mathrm{FE}}=\frac{\left|\ln Z_{\text {exact }}-\ln Z\left(D_{\text {cut }}\right)\right|}{\left|\ln Z_{\text {exact }}\right|},
$$

which clarify the difference between the numerical results with the exact values. We observe that the error decreases monotonically in the range of $0 \leq m \leq 2$ as $D_{\text {cut }}$ increases from 14 to 22 . It is also noted that the error diminishes monotonically as the fermion mass becomes larger. The

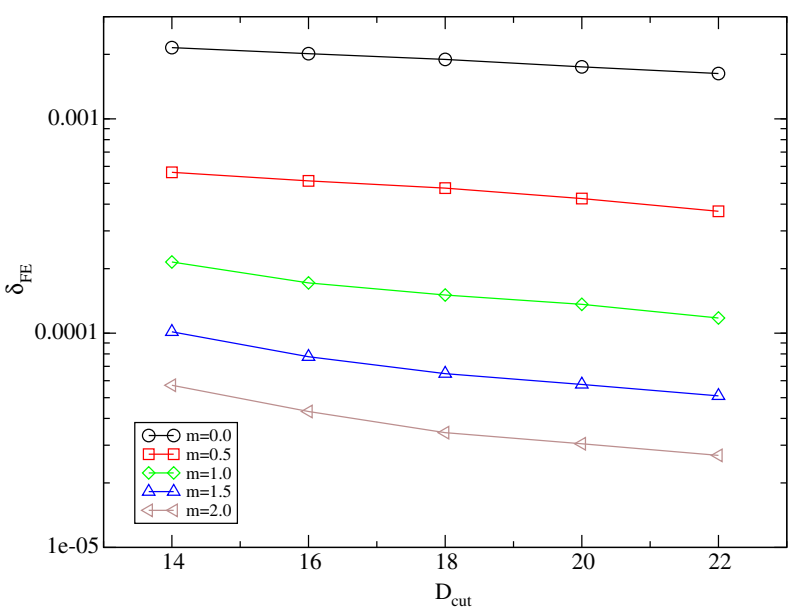

FIG. 8. Error of free energy defined by Eq. (57) as a function of $D_{\text {cut }}$.

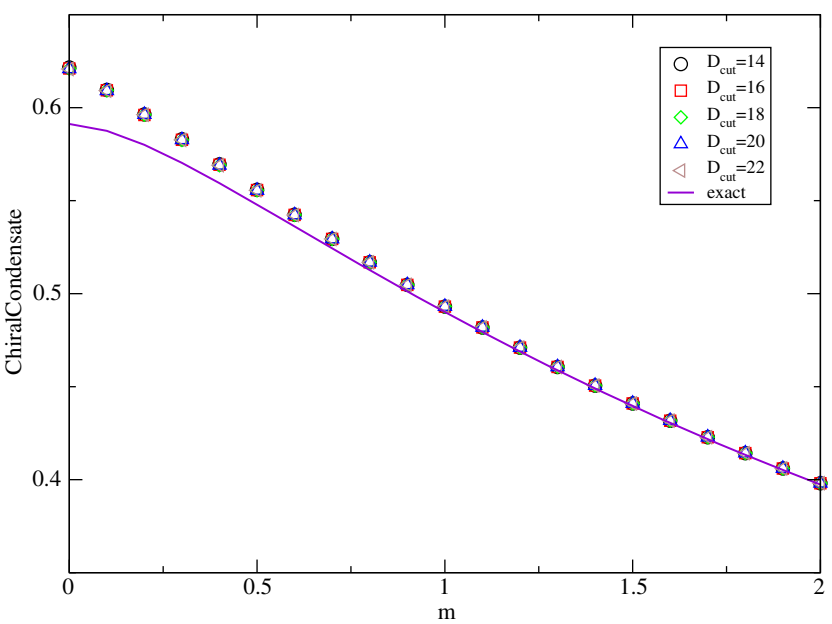

FIG. 9. Chiral condensate as a function of $m$.

maximal error is about $0.2 \%$ at $m=0$ and the minimal one is about $0.02 \%$ at $m=2$. Figure 8 compares the errors as a function of the $D_{\text {cut }}$ for some $m$. The error change most at $m=2.0$ and least at $m=0$ for $D_{\text {cut }}$.

\section{B. Chiral condensate}

We summarize the results for the chiral condensate in Figs. 9 and 10. The former plots the numerical results as a function of the fermion mass $m$ together with the exact values. We find a clear deviation between them near $m=0$. The latter shows the magnitude of the error defined by

$$
\delta_{\mathrm{CC}}=\frac{\left|\langle\bar{\psi} \psi\rangle_{\text {exact }}-\langle\bar{\psi} \psi\rangle\left(D_{\mathrm{cut}}\right)\right|}{\left|\langle\bar{\psi} \psi\rangle_{\mathrm{exact}}\right|} .
$$

The error reduces monotonically as the fermion mass increases. This is a similar tendency as observed for the error of the free energy. The maximal error is about 5\% at

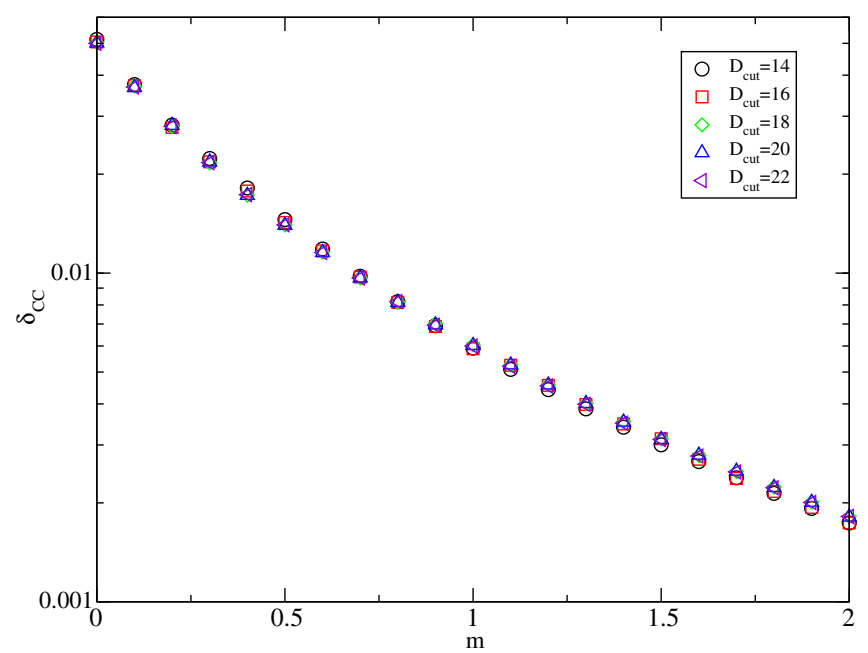

FIG. 10. Error of chiral condensate defined by Eq. (58) as a function of $m$. 
$m=0$ and the minimal one is about $0.1 \%$ at $m=2$. It should be noted that the error does not change so much against the increment of $D_{\text {cut }}$. The reason may be that we use the common unitary matrices, which are optimized for the normal tensors but not for the impure tensors, in the coarse-graining of both tensors.

\section{Fermion two-point correlation functions}

We consider $C_{11}\left(n_{1}, n_{2}\right)$ and $C_{12}\left(n_{1}, n_{2}\right)$ as representative cases. Figures $11,12,13$, and 14 show real and imaginary parts of $C_{11}\left(n_{1}, n_{2}\right)$ and $C_{12}\left(n_{1}, n_{2}\right)$, respectively, as a function of $R$, which is defined by $n_{2}=n_{1}+R \cdot \hat{1}$. In Figs. 11 and 13 , we find that the accuracy of the real part becomes worse on $m=0$ as observed for the free energy and the chiral condensate. Another important finding is that the errors become relatively large at larger $R$ for all the choices of $m$. Here we point out that the magnitude of the imaginary part may give us an estimate of the magnitude of the error for the real

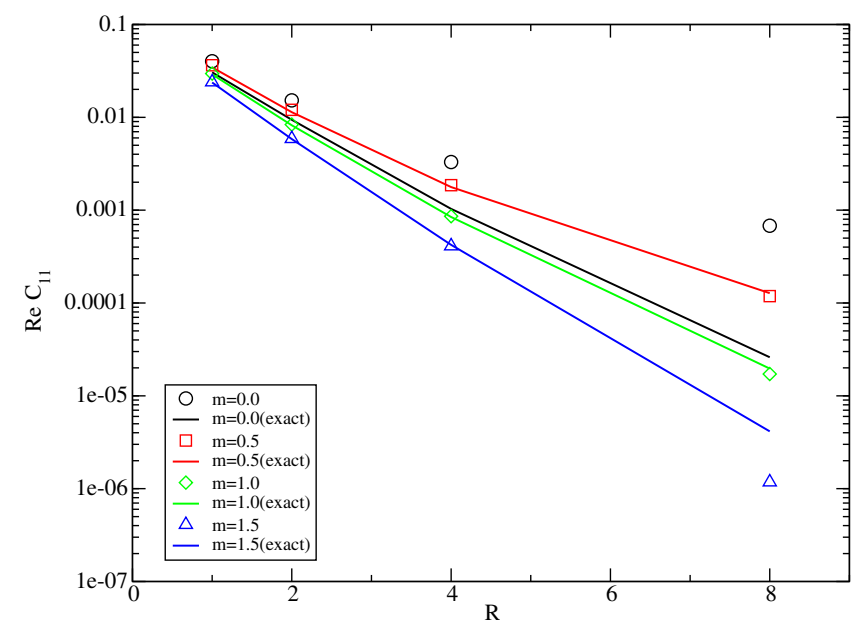

FIG. 11. Real part of $C_{11}\left(n_{1}, n_{2}\right)$.

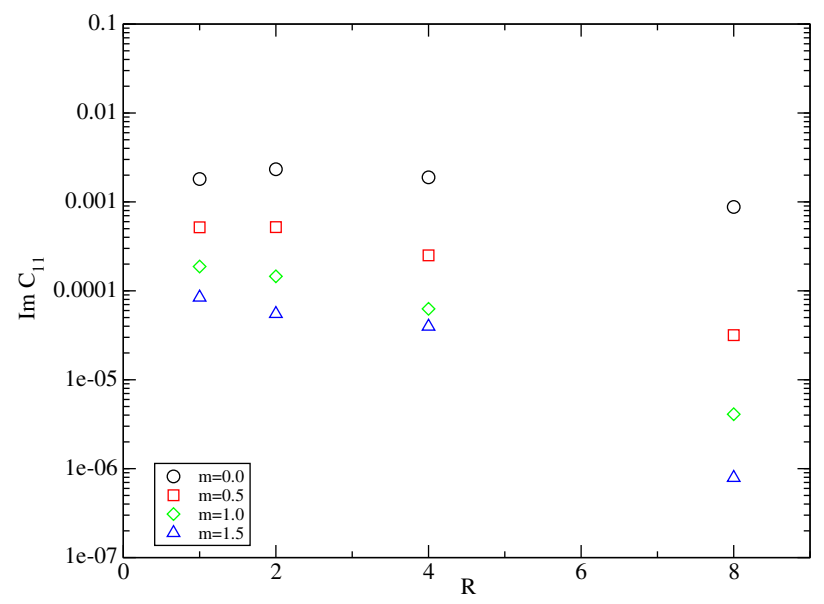

FIG. 12. Imaginary part of $C_{11}\left(n_{1}, n_{2}\right)$.

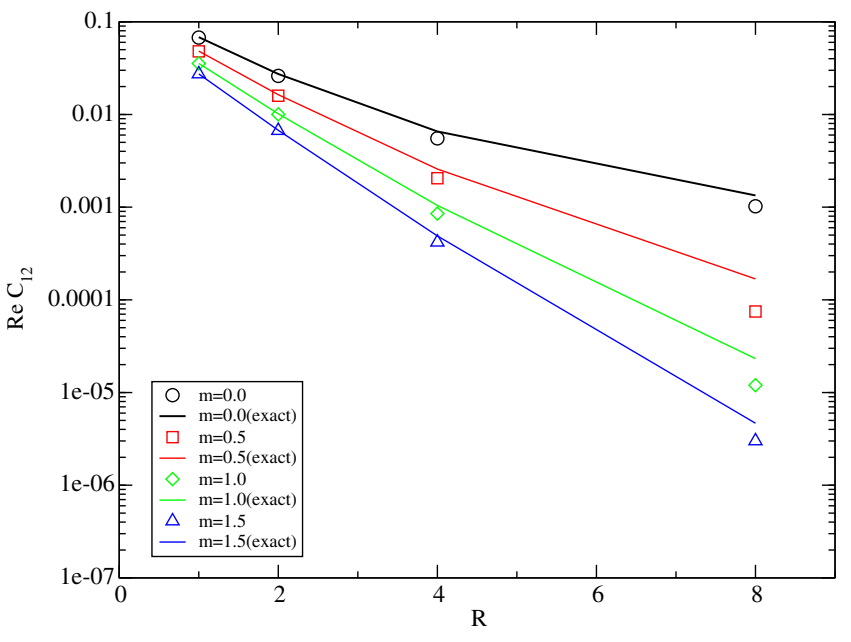

FIG. 13. Real part of $C_{12}\left(n_{1}, n_{2}\right)$.

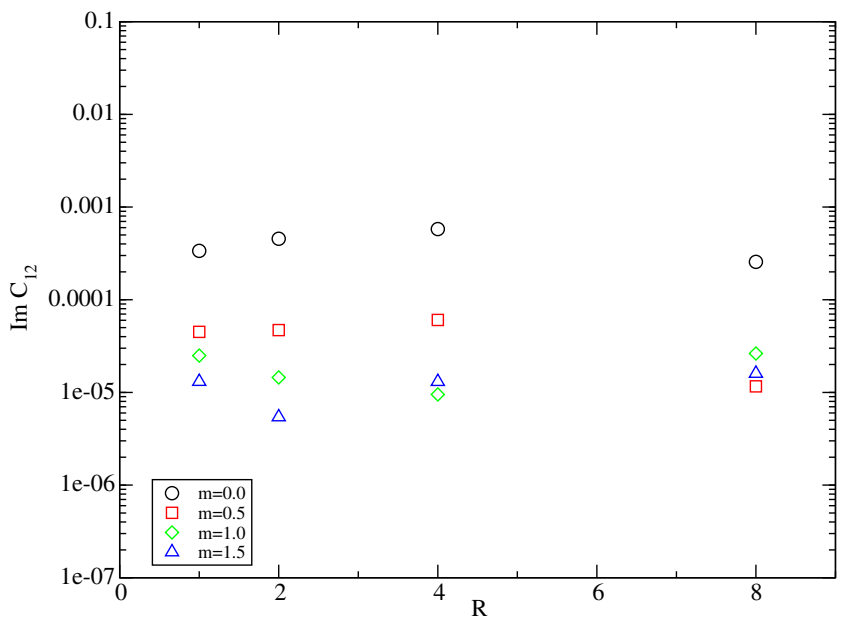

FIG. 14. Imaginary part of $C_{12}\left(n_{1}, n_{2}\right)$.

part, since the imaginary part is expected to vanish theoretically. Figures 12 and 14, in fact, show that the magnitude of the real part at $R=8$ are comparable with that of the imaginary part. This indicates that the signal-tonoise ratio of the real part at the large distance (around $R=8$ ) may be of order of one. In our case, where the correlation length is small, it is difficult to accurately compute the correlation function at large separation. For the case of the larger correlation length, however, one may measure the long separated correlation function, whose signal-to-noise ratio is expected to be better.

\section{SUMMARY AND OUTLOOK}

We have developed a method to calculate fermionic Green functions in the framework of GHOTRG using the impure tensors. The validity of the method is tested by calculating the chiral condensate and the fermion two-point correlation functions in the three-dimensional free Wilson fermion system on a $256^{3}$ lattice, where we employ $0 \leq$ 
$m \leq 2$ for the fermion mass parameter and $14 \leq D_{\text {cut }} \leq 22$ for the tensor indices. In most cases we find good consistency between the numerical results and the exact values: their deviation is at most a few percentage level. The only exception is the fermion two-point correlation functions at a long distance. It may be due to the difficulty of evaluating tiny correlations left at a long distance.

The method developed in this paper can be applied to any physical systems which contain fermions. We plan to apply it to more complicated systems including gauge interactions. It is also important to improve the accuracy of the method. It could be effective to use an idea of the higher-order second renormalization group (HOSRG) $[10,18]$ for the formulation of impure tensors.

\section{ACKNOWLEDGMENTS}

This research used the computational resources of HA-PACS and COMA provided by the Interdisciplinary Computational Science Program in the Center for Computational Sciences, University of Tsukuba. This research is supported by the Ministry of Education, Culture, Sports, Science and Technology (MEXT) as "Exploratory Challenge on Post-K computer (Frontiers of Basic Science: Challenging the Limits)" and also in part by Grants-in-Aid for Scientific Research from MEXT (No. 15H03651 and No. 17K05411).

\section{APPENDIX: BLOCK DIAGONALIZATION}

We explain the block diagonalization for the bosonic tensors. Let us consider the following matrix representation for a given bosonic tensor,

$$
T_{r, c}^{\text {matrix }}=T_{x_{1, n} x_{2, n} x_{3, n} x_{1, n-1} x_{2, n-\hat{2}} x_{3, n-\hat{3}}},
$$

with

$$
\begin{gathered}
r=x_{1, n} \otimes x_{2, n} \otimes x_{3, n}, \\
c=x_{1, n-\hat{1}} \otimes x_{2, n-\hat{2}} \otimes x_{3, n-\hat{3}} .
\end{gathered}
$$

Thanks to the condition of Eq. (9), the matrix can be blockdiagonalized as

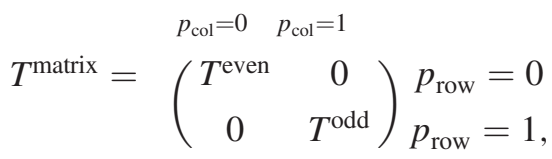

with

$$
\begin{aligned}
p_{\text {row }} & =\left(\sum_{i=1}^{3} \sum_{s=1}^{2} x_{i, n, s}\right) \bmod 2, \\
p_{\text {col }} & =\left(\sum_{i=1}^{3} \sum_{s=1}^{2} x_{i, n-\hat{i}, s}\right) \bmod 2 .
\end{aligned}
$$

Similarly, the contracted tensor of Eq. (10) can also be block-diagonalized. Thus, the eigenvalue decomposition of Eq. (16) is independently applied to the even and odd blocks yielding the associated unitary matrices $U_{i}^{\text {even }}$ and $U_{i}^{\text {odd }}$. The block-diagonalized unitary matrix is expressed as

$$
\begin{aligned}
& x_{i, n^{*}, \mathrm{f}}=0 \quad x_{i, n^{*}, \mathrm{f}=1} \\
& U_{i}^{\text {matrix }}=\left(\begin{array}{cc}
U_{i}^{\text {even }} & 0 \\
\underbrace{0} & \underbrace{U_{i}^{\text {odd }}}
\end{array}\right) \begin{array}{l}
p_{n}=0 \\
p_{n}=1,
\end{array} \\
& D_{\text {even }} \quad D_{\text {odd }}
\end{aligned}
$$

with

$$
p_{n}=\left(\sum_{s=1}^{2} x_{i, n, s}+x_{i, n-\hat{i}, s}\right) \bmod 2,
$$

where $D_{\text {even }}+D_{\text {odd }}=D_{\text {cut }}$.

This property can be used to reduce the computational and memory costs by dealing with only nontrivial blocks. Moreover, the blocks satisfy the condition of Eq. (28) so that it is not necessary to extend the fermionic indices for the bosonic tensor as Eq. (31).
[1] M. Levin and C. P. Nave, Phys. Rev. Lett. 99, 120601 (2007).

[2] L. P. Kadanoff II, I1 Nuovo Cimento B (1965-1970) 44, 276 (1966).

[3] Z.-C. Gu, F. Verstraete, and X.-G. Wen, arXiv: 1004.2563; Z.-C. Gu, Phys. Rev. B 88, 115139 (2013).

[4] Y. Shimizu and Y. Kuramashi, Phys. Rev. D 90, 014508 (2014).

[5] Y. Shimizu, Chin. J. Phys. 50, 749 (2012).
[6] Y. Shimizu and Y. Kuramashi, Phys. Rev. D 90, 074503 (2014).

[7] S. Takeda and Y. Yoshimura, Prog. Theor. Exp. Phys. 2015, 043B01 (2015).

[8] G. Evenbly and G. Vidal, Phys. Rev. Lett. 115, 180405 (2015).

[9] S. Yang, Z.-C. Gu, and X.-G. Wen, Phys. Rev. Lett. 118, 110504 (2017).

[10] Z. Y. Xie, J. Chen, M. P. Qin, J. W. Zhu, L. P. Yang, and T. Xiang, Phys. Rev. B 86, 045139 (2012). 
[11] S. Wang, Z.-Y. Xie, J. Chen, N. Bruce, and T. Xiang, Chin. Phys. Lett. 31, 070503 (2014).

[12] J. F. Yu, Z. Y. Xie, Y. Meurice, Y. Liu, A. Denbleyker, H. Zou, M. P. Qin, J. Chen, and T. Xiang, Phys. Rev. E 89, 013308 (2014).

[13] J. Unmuth-Yockey, Y. Meurice, J. Osborn, and H. Zou, arXiv:1411.4213.

[14] H. Kawauchi and S. Takeda, Phys. Rev. D 93, 114503 (2016).
[15] R. Sakai, S. Takeda, and Y. Yoshimura, Prog. Theor. Exp. Phys. 2017, 063B07 (2017).

[16] Z. C. Gu, M. Levin, and X. G. Wen, Phys. Rev. B 78, 205116 (2008).

[17] N. Nakamoto and S. Takeda, Sci. Rep. Kanazawa Univ. 60, 11 (2016).

[18] Z. Y. Xie, H. C. Jiang, Q. N. Chen, Z. Y. Weng, and T. Xiang, Phys. Rev. Lett. 103, 160601 (2009). 